\title{
Real-world data on the efficacy and safety of daratumumab treatment in Hungarian relapsed/refractory multiple myeloma patients
}

\author{
Szilvia Lovas ${ }^{1} \cdot$ Gergely Varga $^{2} \cdot$ Péter Farkas $^{2} \cdot$ Tamás Masszi $^{2} \cdot$ Nikolett Wohner $^{2} \cdot$ Ágnes Bereczki $^{3}$. \\ Nóra Adamkovich ${ }^{3} \cdot$ Zita Borbényi $^{3}$ - Árpád Szomor ${ }^{4}$. Hussain Alizadeh ${ }^{4} \cdot$ Erika Szaleczky $^{5} \cdot$ Krisztina Wolf $^{5}$. \\ Tamás Schneider ${ }^{5} \cdot$ Márk Plander $^{6} \cdot$ Tamás Szendrei $^{6}$. Ottó Csacsovszki ${ }^{7}$. Zoltán Csukly ${ }^{7} \cdot$ Péter Rajnics $^{8}$. \\ Miklós Egyed ${ }^{8} \cdot$ Zsolt Nagy $^{9} \cdot$ László Rejtö $^{10} \cdot$ Árpád Illés ${ }^{1} \cdot$ Gábor Mikala $^{7} \cdot$ László Váróczy $^{1}$ (1)
}

Received: 8 May 2019 / Revised: 2 August 2019 / Accepted: 2 August 2019

(c) Japanese Society of Hematology 2019

\begin{abstract}
Daratumumab is a human anti-CD38 monoclonal antibody used in the treatment of refractory and relapsed multiple myeloma. We investigated the efficacy and safety of daratumumab therapy in a real-world setting. Ninety-nine Hungarian patients were included; 48 received monotherapy, while lenalidomide and bortezomib combinations were administered in 29 and 19 cases, respectively. Overall response rate was assessable in 88 patients, with 12 complete, 10 very good partial, 34 partial, and seven minor responses. At a median duration of follow-up of 18.6 months, median progression-free survival (PFS) among all patients was 17.0 months. These values were inferior in the bortezomib combination and monotherapy groups. Patients with early-stage disease (ISS1) had better survival results than those with stage 2 or 3 myeloma $(p=0.009)$. Heavily pretreated patients had inferior PFS compared to those with 1-3 therapies $(p=0.035)$. Patients with impaired renal function had PFS results comparable with those having no kidney involvement. There were 10 fatal infections, and the most frequent adverse events were mild infusion-associated reactions and hematologic toxicities. Our results confirm that daratumumab is an effective treatment option for relapsed/refractory MM with an acceptable safety profile in patients with normal and impaired renal function.
\end{abstract}

Keywords Multiple myeloma $\cdot$ Daratumumab $\cdot$ Treatment response $\cdot$ Survival $\cdot$ Toxicity

Gábor Mikala and László Váróczy equally contributed to this work.

László Váróczy

varoczy@internal.med.unideb.hu

1 Department of Hematology, Institute for Medicine, Faculty of Medicine, University of Debrecen, Nagyerdei krt. 98., 4032 Debrecen, Hungary

23 rd Department of Internal Medicine, Semmelweis University, Budapest, Hungary

3 2nd Department of Internal Medicine and Cardiology Center, Faculty of Medicine, University of Szeged, Szeged, Hungary

4 1st Department of Internal Medicine, University of Pécs, Pecs, Hungary

5 National Institute of Oncology, Budapest, Hungary

\section{Introduction}

Multiple myeloma (MM) is a malignant hematologic disorder that accounts for one percent of all cancer cases. It can be characterized by clonal proliferation of plasma cells; its main complications include the development of

6 Department of Hematology, Markusovszky Teaching Hospital, Szombathely, Hungary

7 Department of Hematology and Stem Cell Transplantation, South-Pest Central Hospital, National Institute for Hematology and Infectious Diseases, Budapest, Hungary

8 Department of Hematology, Mór Kaposi Teaching Hospital, Kaposvár, Hungary

9 1st Department of Internal Medicine, Semmelweis University, Budapest, Hungary

10 Department of Hematology, Jósa András Teaching Hospital, Nyíregyháza, Hungary 
lytic bone lesions, hypercalcaemia, bone marrow failure, and renal impairment. It is considered as an incurable disease; however, there has been a significant improvement in patients' survival, thanks to the novel drugs that have been recently introduced [1]. Besides immunomodulatory drugs (IMID) and proteasome inhibitors (PI), monoclonal antibodies $(\mathrm{moAB})$ have also become available for the treatment of refractory-relapsing cases. One of the important antibody targets in MM is CD38 that is highly expressed on plasma cells. However, CD38 can be also detected on the surface of NK-cells, activated T cells, B cells, erythrocytes, and platelets [2]. Daratumumab is a first-in-class, human

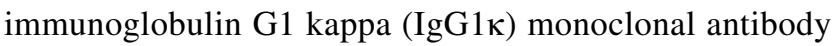
that targets CD38 and kills plasma cells through antibodydependent cellular cytotoxicity- and complement-dependent cytotoxicity-mediated mechanisms [3]. In addition to these immune-mediated killing processes, daratumumab may also have an immunomodulatory role via $\mathrm{T}$ cell activation and expansion as well as moderation of immunosuppression. The anti-tumor efficacy of daratumumab can be increased by the addition of immunomodulatory drugs. Lenalidomide can facilitate antibody-mediated lysis of plasma cells by activating autologous effector cells within the natural environment of the tumor [4]. This conclusion was confirmed by another study where daratumumab was combined with lenalidomide and bortezomib [5].

Currently, daratumumab is one of the most effective new agents lately approved for relapsed/refractory multiple myeloma (RRMM), first in monotherapy in massively pretreated patients, later in triplet combinations from the first relapse. Although clinical trials showed good tolerability and high effectivity of daratumumab both in monotherapy and as combined with lenalidomide or bortezomib, trials are different from real-world use for many reasons including less rigorous patient selection, greater flexibility with dosing and combinations, and also country-specific funding restrictions making real-world data very important for clinicians. In terms of daratumumab, the registration studies limited the inclusion to patients with normal or only moderately altered renal function and real-world data regarding daratumumab use in patients with renal failure is currently very limited.

The aim of this study was to evaluate the efficacy and safety of daratumumab-based therapies in a large cohort of Hungarian relapsed/refractory MM patients.

\section{Patients, procedures, and methods}

We approached all centers in Hungary where daratumumab was used, in a retrospective data collection. The clinical files of multiple myeloma patients were reviewed with particular reference to age, sex, clinical stage, renal function, response to treatment and survival. ISS stages were determined using the International Myeloma Working Group (IMWG) criteria. The way how FISH testing was performed varied, there was no consensus regarding the probes used but those for $17 \mathrm{p}$ deletion, translocations $(11 ; 14),(4 ; 14)$ and $(14 ; 16)$, and $1 \mathrm{q}$ amplification were generally part of the set. FISH results of unfavorable prognosis included $\mathrm{t}(4 ; 14), \mathrm{t}(14 ; 16)$, and $\operatorname{del}(17 \mathrm{p})$.

Patients received daratumumab in the dose of $16 \mathrm{mg} / \mathrm{kg}$ intravenously once weekly in the first 8 weeks, than $16 \mathrm{mg} /$ $\mathrm{kg}$ every second week (lenalidomide arm) or $16 \mathrm{mg} / \mathrm{kg}$ every third week (bortezomib arm) for 12 weeks and finally $16 \mathrm{mg} /$ $\mathrm{kg}$ every 4 weeks. $20 \mathrm{mg}$ dexamethasone was given orally or intravenously on days $1,2,4,5,8,9,11$, and 12. Bortezomib was administered at doses varying from 1 to $1.3 \mathrm{mg} / \mathrm{m}^{2}$ on days $1,4,8,11$ or only on days 1 and 8 , while lenalidomide was given at doses of 10-25 mg on days 1-21. Response criteria (complete response [CR], very good partial response [VGPR], partial response [PR], no response [NR], and progressive disease $[\mathrm{PD}]$ ) and survival measures (progressionfree survival [PFS] and overall survival [OS]) were defined according to the published IMWG guidelines. Examining the survival rates, overall survival (OS) was determined by consideration of death events due to any reasons, while progression-free survival (PFS) was determined by consideration of relapses, death events or disease progression that indicated further treatment. Survival rates were calculated using the Kaplan-Meier's method, while the survival data were compared using the log-rank test. Differences were significant if probability level was less than $5 \%(p<0.05)$.

\section{Results}

\section{Patient characteristics}

Ten centers responded with 99 patients altogether, who were treated with daratumumab-based therapies between September 2016 and December 2018. Patient demographics were generally balanced. $51.1 \%$ of the patients were male. Average age was $60.3 \pm 10.7$ (median: 62; range 28-84). Patients were heavily pretreated, the median number of prior lines was 3 (range 1-12). Almost all patients had prior bortezomib (97\%) and most had lenalidomide (77.8\%) and thalidomide (82.8\%), all but one had one of the two IMiDs. $62.6 \%$ of the patients had prior autologous transplantation, two prior allograft as well. Pre-daratumumab cytogenetic FISH tests of 63 patients were available, and here $36(57.1 \%)$ had high risk cytogenetics. The majority had high International Staging System (ISS) Score: 20, 19, and 60 were in the ISS 1, 2 , and 3 groups, respectively. Treatment usually continued until progression, unacceptable toxicity or death, the median number of cycles was 5. Markedly impaired renal function 
(GFR $<30 \mathrm{ml} / \mathrm{min}$ ) has been reported in 11 cases, including 6 patients who required hemodialysis (Table 1).

\section{Adverse events}

According to the reported number of adverse events (AEs), daratumumab treatment was well tolerated. AEs above grade 1-2 were rare. The most frequent AEs were mild infusionassociated reactions (IARs, grade 1-2), hematologic toxicities (mostly neutropenia), and infections.

There were ten fatal infections that included 6 cases with septicaemia and 4 cases with bronchopneumonia. Other fatal AEs were absent. Infusion-related toxicities were manageable in all cases (Table 2).

Table 1 Clinical characteristics of patients

\begin{tabular}{|c|c|}
\hline Characteristics & \\
\hline \multicolumn{2}{|l|}{ Age at the diagnosis, years } \\
\hline Median (range) & $62(28-84)$ \\
\hline \multicolumn{2}{|l|}{ Distribution, $n$} \\
\hline$<65$ & 62 \\
\hline $65-74$ & 31 \\
\hline $75 \leq$ & 6 \\
\hline Median time from diagnosis, years (range) & $4(0-27)$ \\
\hline \multicolumn{2}{|l|}{ Type of measurable disease, $n(\%)$} \\
\hline $\operatorname{IgG}$ & $53(53.5)$ \\
\hline $\operatorname{IgA}$ & $19(19.2)$ \\
\hline Light chain & $27(27.3)$ \\
\hline \multicolumn{2}{|l|}{ ISS disease staging, $n(\%)$} \\
\hline I & $20(20.2)$ \\
\hline II & $19(19.2)$ \\
\hline III & $60(60.6)$ \\
\hline \multicolumn{2}{|l|}{ Number of prior lines of therapy (\%) } \\
\hline Median (range) & $3(1-12)$ \\
\hline $1-3$ & $43(43.4)$ \\
\hline$>3$ & $56(56.6)$ \\
\hline \multicolumn{2}{|l|}{ Prior treatments, $n(\%)$} \\
\hline Bortezomib & $96(97)$ \\
\hline Thalidomide & $82(82.8)$ \\
\hline Lenalidomide & $77(77.8)$ \\
\hline Prior ASCT, $n(\%)$ & $61(62.6)$ \\
\hline \multicolumn{2}{|l|}{ Pre-daratumumab cytogenetic profile, $n(\%)$} \\
\hline Standard-risk cytogenetic abnormality & $17 / 63(27)$ \\
\hline High-risk cytogenetic abnormality & $36 / 63(57.1)$ \\
\hline dell $7 p$ & $7 / 63(11.1)$ \\
\hline $\mathrm{t}(4,14)$ & $8 / 63(12.7)$ \\
\hline $\mathrm{t}(14,16)$ & $2 / 63(3.2)$ \\
\hline Other & $10 / 63(15.9)$ \\
\hline \multicolumn{2}{|l|}{ Renal function, $n(\%)$} \\
\hline Normal or slightly impaired (GFR > $30 \mathrm{ml} / \mathrm{min}$ ) & $88(88.8)$ \\
\hline Markedly impaired (GFR <30 ml/min) & $11(11.1)$ \\
\hline
\end{tabular}

Table 2 Side effects of daratumumab-based therapies

\begin{tabular}{lrrrc}
\hline & Grade 1-2 & Grade 3 & Grade 4 & Grade 5 \\
\hline Infusion-associated reaction & 15 & & 1 & \\
Neutropenia & 5 & 2 & & \\
Anemia & 1 & 2 & & \\
Thrombocytopenia & & 2 & 1 & \\
Infection & 3 & 4 & 1 & 10 \\
Cardiovascular & 1 & & & \\
Gastroenterology & 2 & & & \\
Neurology & & 1 & 1 & \\
All & 27 & 11 & 4 & 10 \\
\hline
\end{tabular}

\section{Efficacy}

The majority of patients (48 cases, $48.9 \%$ ) had monotherapy with dexamethasone only, but 19 of them (19.3\%) had bortezomib and 29 of them (29.3\%) received lenalidomide in combination. In three cases, daratumumab was combined with other agents (carfilzomib or cyclophosphamide). Patients who received only daratumumab monotherapy underwent more prior treatment lines than those who received combinations with lenalidomide and dexamethasone (DRd) or bortezomib and dexamethasone (DVd); the mean values were $4.13 \pm 2.017,3.58 \pm 2.23$, and $2.77 \pm 0.869$, respectively. Overall response rate was assessable in 88 patients with 12 complete, 10 very good partial, 34 partial, 7 minor responses. 25 showed progressive disease. 9 patients had no formal disease reassessment due to either early death or short follow-up. Those patients who received either bortezomib or lenalidomide combinations showed significantly better overall response rates than those who had monotherapy $(p<0.001)$ (Fig. 1).

The clinical cutoff date was December 31, 2018. At a median duration of follow-up of 18.6 months (range 1-27.5), the median PFS was 17.0 months in the whole cohort. There was a trend of inferior PFS in the bortezomib combination and monotherapy groups (6.6 months in both), while median PFS was not reached in the lenalidomide group (Fig. 2). Patients having an early-stage disease (ISS1) had significantly better survival results than those who had stage 2 or 3 myeloma (Fig. 3). The number of prior therapies seemed to be another important prognostic marker as extensively pretreated patients who received more than 3 treatment lines had inferior PFS data (Fig. 4). In terms of the FISH results, patients having adverse genetic alterations showed markedly inferior survival in comparison with standard FISH results (post hoc analysis, $p=0.049$ ) (Fig. 5). Interestingly, patients with markedly impaired renal function had comparable PFS results to those having no kidney dysfunction (Fig. 6). 
Fig. 1 Therapeutic responses after daratumumab monotherapy, bortezomib and lenalidomide combinations

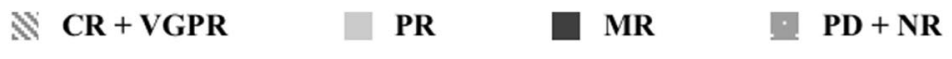

\section{Response}

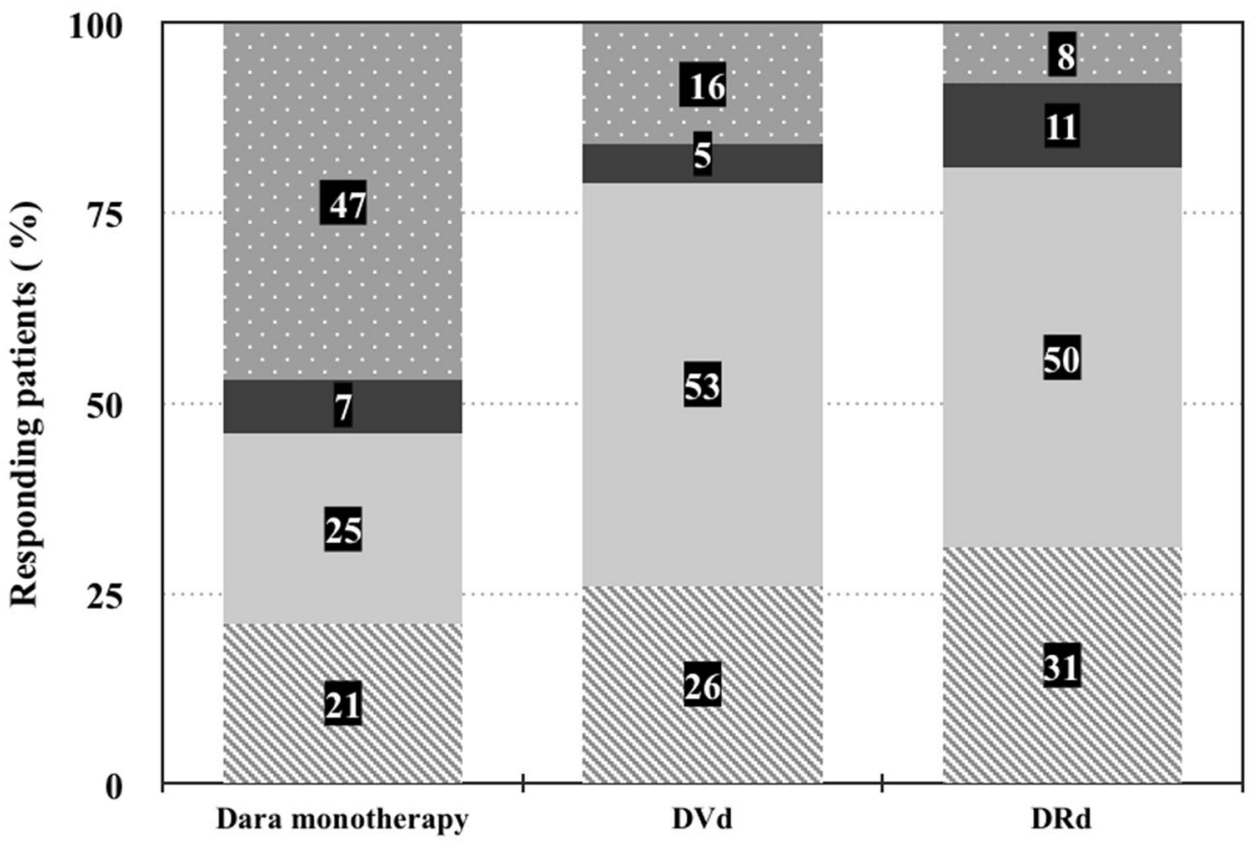

Fig. 2 Progression-free survival in terms of combination regimens

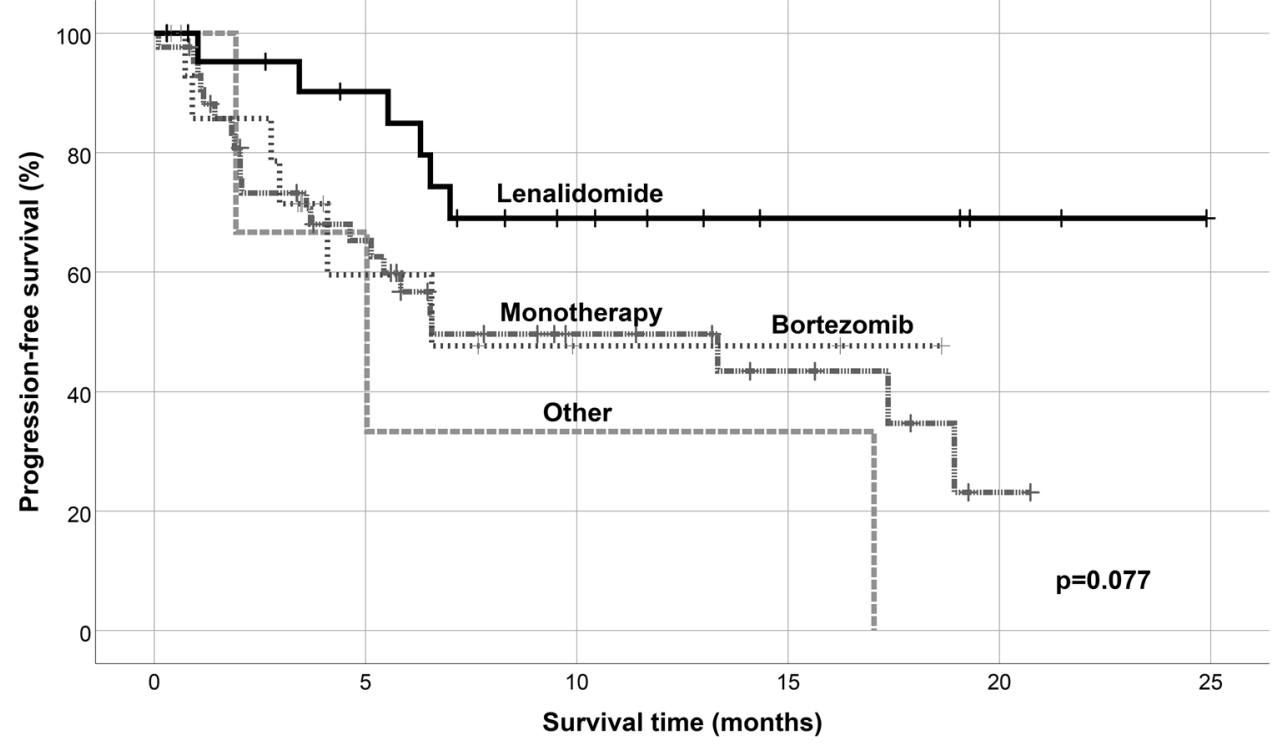

\section{Discussion}

Daratumumab has been recently approved for the treatment of RR multiple myeloma patients. As the first moAB therapy in $\mathrm{MM}$, it has potent single-agent activity, as it was revealed by the GEN501 and SIRIUS trials. Pooled analysis of these studies showed an overall response rate of $31.1 \%$, a median PFS of 4 months, and OS of 20.1 months in RR MM patients with a median of five prior lines of therapy $[6,7]$. Later on, there were two pivotal clinical trials conducted, where daratumumab showed its unique efficacy in combination therapies as well. In the CASTOR trial, daratumumab plus bortezomib and dexamethasone (DVd) treatment was administered for RR MM patients, while bortezomib and dexamethasone were given on the control arm. After 19.4 months of median followup, patients receiving daratumumab-based combination showed significantly more favorable overall response rates $(83.8 \%$ vs. $63.2 \%, p<0.0001)$ and progression-free 
Fig. 3 Progression-free survival in terms of ISS stages

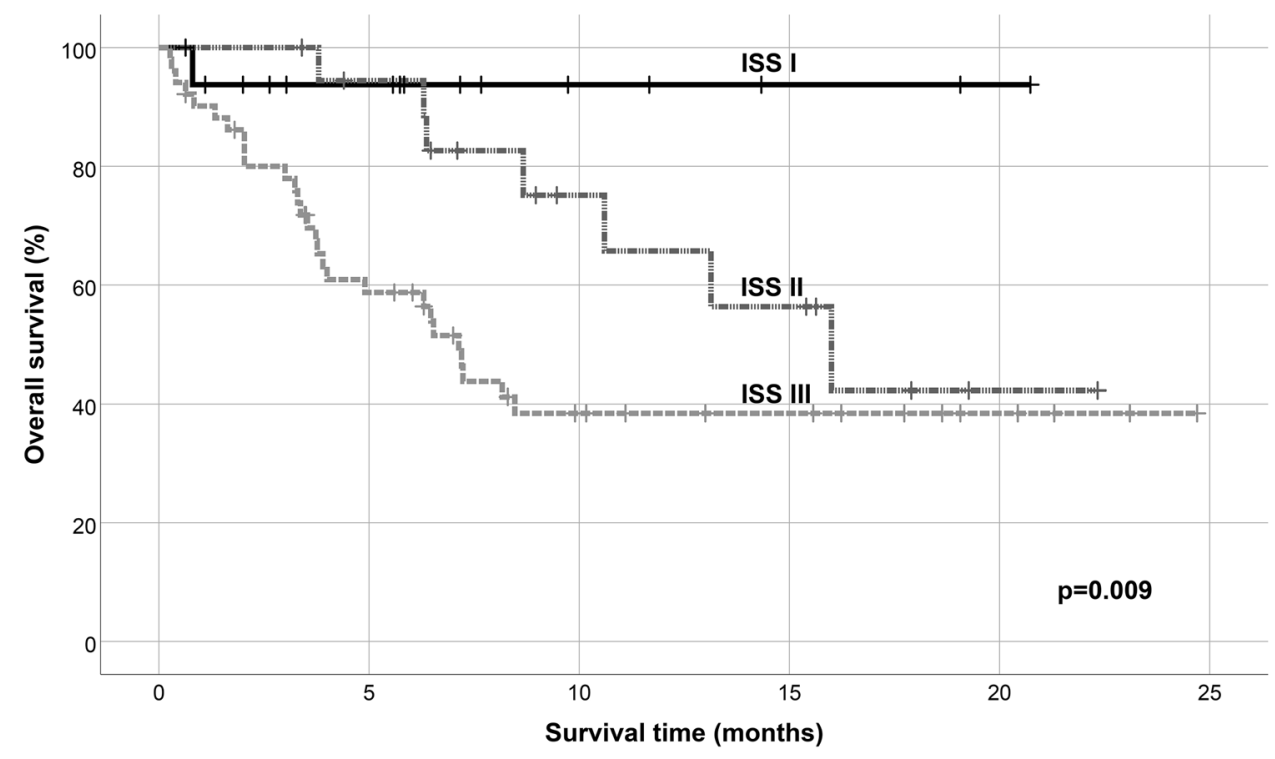

Fig. 4 Progression-free survival in terms of the number of previous treatment lines

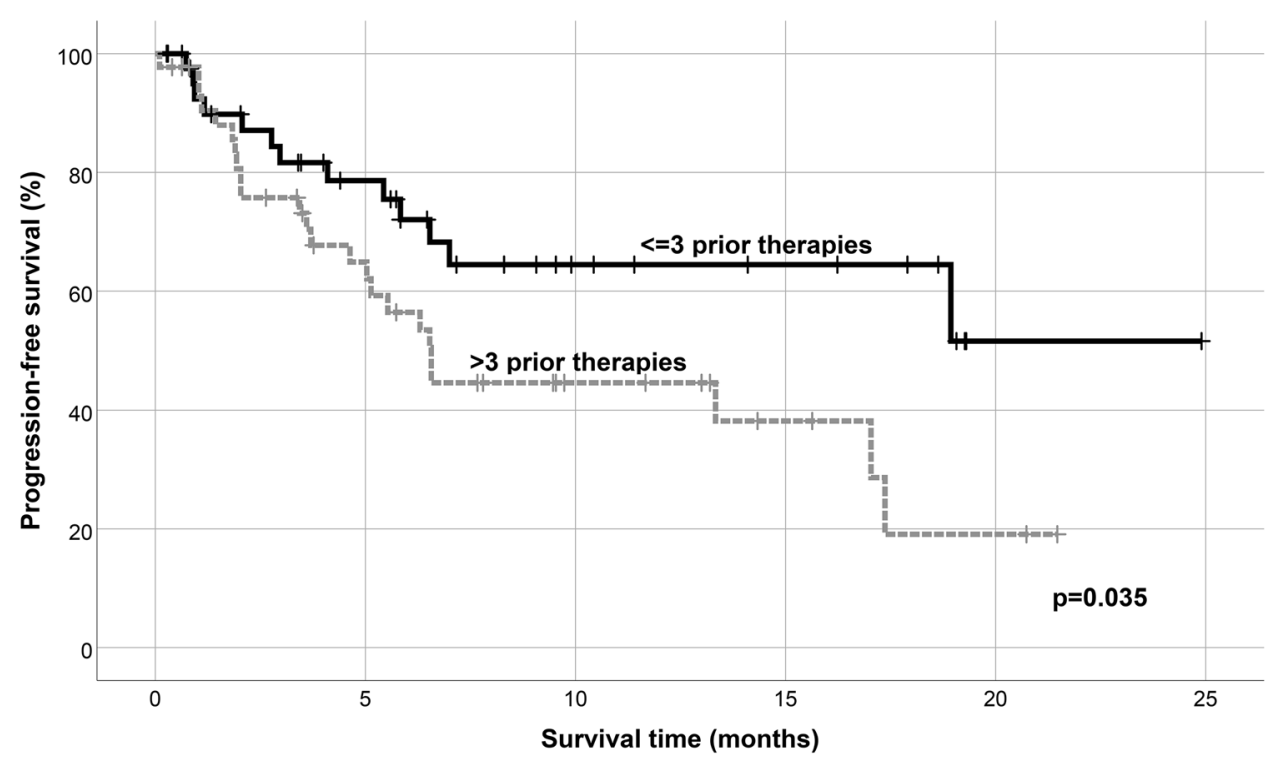

survival (median 16.7 vs. 7.1 months, $p<0.0001$ ) than those being randomized to the control arm. Daratumumab plus bortezomib and dexamethasone was superior in subgroups based on treatment exposure, lenalidomide-refractory status or cytogenetic status $[8,9]$. In the POLLUX trial, patients received either daratumumab plus lenalidomide and dexamethasone (DRd) therapy or lenalidomide and dexamethasone (Len/Dex) combination. After 25.4 months of median follow-up, the DRd treatment prolonged progression-free survival vs. Len/Dex (median not reached vs. 17.5 months, $p<0.0001$ ). The overall response rate was $92.9 \%$ vs. $76.4 \%$, and $51.2 \%$ vs. $21.0 \%$ achieved a complete response $(p<0.0001)$. Analyses of clinically relevant patient subgroups demonstrated that PFS results were favorable for the daratumumab arm even within bortezomib-refractory patients, within those with previous lenalidomide exposure or within those with adverse FISH results $[10,11]$.

There are only a limited number of publications available in terms of real-world results of daratumumab treatment. Minarik et al. reported on 14 RR MM patients who received daratumumab monotherapy after a median 4.5 previous lines. The overall response rate was $38.5 \%$. The median progression-free survival was 4.6 months and median overall survival was not achieved. The toxicities were mostly mild [12]. A French group reported the results of a single-center series of 41 RRMM patients treated with single-agent daratumumab outside clinical trials. Patients received a median number of 4 prior therapies. All patients were previously exposed to PI and IMID and all patients were refractory to 
Fig. 5 Progression-free survival in terms of FISH results

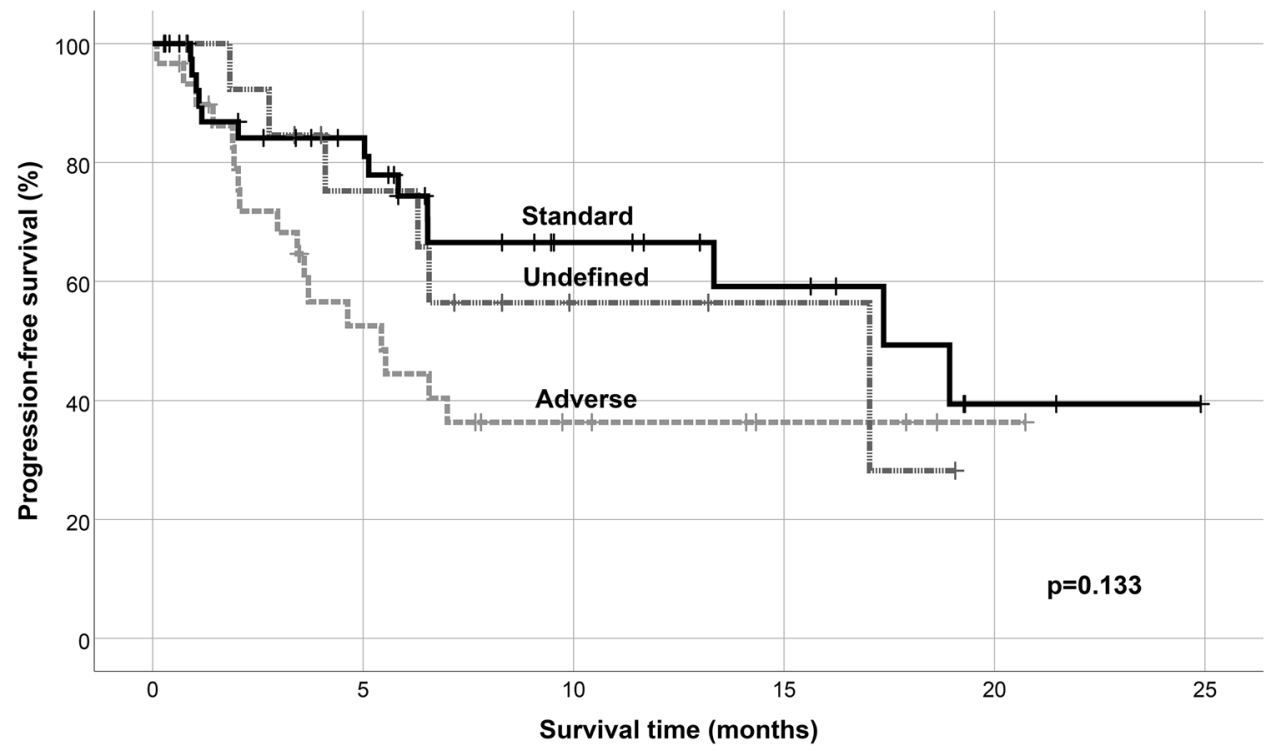

Fig. 6 Progression-free survival in terms of renal function

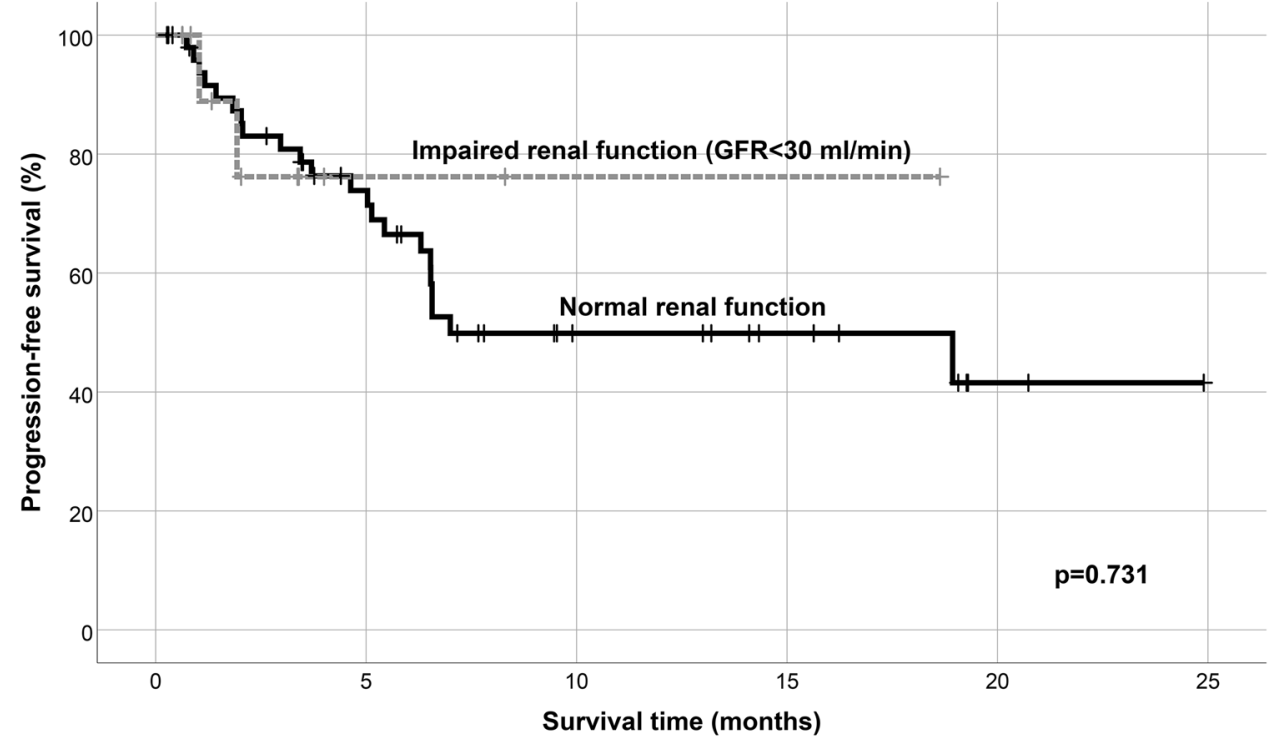

the last line of therapy. The overall response rate was $24 \%$; however, after a median follow-up of 6.5 months, all patients experienced disease relapse. The median progression-free survival was only 1.9 months [13].

In Hungary, ninety-nine RR multiple myeloma patients received daratumumab therapy outside any clinical trials until December 2018. Our treatment protocols were strongly influenced by funding rules of the National Health Insurance, since daratumumab had been reimbursed only as monotherapy for patients who received over 3 prior lines of treatment until June 2017 and then it became available as a part of combination therapy for patients who received $>2$ prior treatment modalities. As a result, most of our patients were heavily pretreated and received only daratumumab monotherapy. Their treatment responses and survival results were still comparable to the data of the SIRIUS trial. However, those patients who received either bortezomib or lenalidomide combination therapies showed inferior treatment and survival results compared to the data known from the CASTOR and POLLUX trials. This can be explained by the fact that our patients came from the "real world" and no strict exclusion criteria were applied regarding the complete blood count, renal function, and ECOG status. Moreover, we treated much less 2 nd and 3rd line patients who dominated the POLLUX and CASTOR trials [14].

Analyzing the data of relevant subgroups, we detected that ISS and the number of prior therapies were the most important predictive factors in terms of the survival results. Patients having adverse genetic alterations showed markedly inferior survival compared to those with standard FISH 
results. Interestingly, patients with impaired renal function showed similar PFS results to those who had normal GFR values. As this population was excluded from the POLLUX and CASTOR trials, we are the first to demonstrate that daratumumab can be safely administered for MM patients with renal failure, including those on dialysis.

Serious adverse events including patients' deaths occurred in 14 cases (14.1\%) which rate is also higher than those observed in the official clinical trials. Fortunately, most of our patients developed no or only minor side effects.

We confirmed in a real-world setting that daratumumab is an effective treatment option for relapsed/refractory MM with acceptable safety profile; however, not surprisingly, our treatment results and survival data were inferior to the data of selected patients of official clinical trials. Our study is the first one that reports real-world results of daratumumab treatment in the settings of monotherapy and combinations as well. Moreover, we are the first to demonstrate that daratumumab can be safely used in a large cohort of patients with GFR $<30 \mathrm{ml} / \mathrm{min}$.

Funding No funding is applicable.

\section{Compliance with ethical standards}

Conflict of interest The authors declare that they have no conflict of interest.

\section{References}

1. Raza S, Safyan RA, Rosenbaum E, Bowman A, Lentzsch S. Optimizing current and emerging therapies in multiple myeloma: a guide for the haematologist. Ther Adv Hematol. 2017;8:55-70.

2. Morandi F, Horenstein AL, Costa F, Guiliani N, Pistoia V, Malavasi F. CD38: a target for immunotherapeutic approaches in multiple myeloma. Front Immunol. 2018;9:2722. https://doi. org/10.3389/fimmu.2018.02722 (eCollection).

3. de Weers M, Tai YT, van der Veer MS, Bakker JM, Vink T, Jacobs DC, et al. Daratumumab, a novel therapeutic human CD38 monoclonal antibody, induces killing of multiple myeloma and other hematological tumors. J Immunol. 2011;186(3):1840-8.

4. Van der Weer MS, De Weers M, Van Kessel B, Bakker JM, Wittebol S, Parren PW, et al. Towards effective immunotherapy of myeloma: enhanced elimination of myeloma cells by combination of lenalidomide with the human CD38 monoclonal antibody daratumumab. Hematologica. 2011;96:284-90.

5. Nijhof IS, Groen RW, Noort WA, van Kessel B, de Jong-Korlaar $\mathrm{R}$, Bakker J, et al. Preclinical evidence for the therapeutic potential of CD28-targeted immune-chemotherapy in multiple myeloma patients refractory to lenalidomide and bortezomib. Clin Cancer Res. 2015;21:2802-10.

6. Lonial S, Weiss BM, Usmani SZ, Singhal S, Chari A, Bahlis NJ, et al. Daratumumab monotherapy in patients with treatmentrefractory multiple myeloma (SIRIUS): an open-label, randomized, phase 2 trial. Lancet. 2016;387:1551-60.

7. Usmani SZ, Weiss BM, Plesner T, Bahlis NJ, Belch A, Lonial S, et al. Clinical efficacy of daratumumab monotherapy in patients with heavily pretreated relapsed or refractory multiple myeloma. Blood. 2016;128:37-44.

8. Palumbo A, Chanan-Khan A, Weisel K, Nooka AK, Masszi T, Beksac M, et al. Daratumumab, bortezomib and dexamethasone for multiple myeloma. N Engl J Med. 2016;375:754-66.

9. Spencer A, Lentzsch S, Weisel K, Avet-Loiseau H, Mark TM, Spicka I, et al. Daratumumab plus bortezomib and dexamethasone versus bortezomib and dexamethasone in relapsed or refractory multiple myeloma: updated analysis of CASTOR. Haematologica. 2018;103(12):2079-87.

10. Dimopoulos MA, Oriol A, Nahi H, San-Miguel J, Bahlis NJ, Usmani SZ, et al. Daratumumab, lenalidomide and dexamethasone for multiple myeloma. N Engl J Med. 2016;375:1319-31.

11. Dimopoulos MA, San-Miguel J, Belch A, White D, Benboubker L, Cook G, et al. Daratumumab plus lenalidomide and dexamethasone versus lenalidomide and dexamethasone in relapsed or refractory multiple myeloma. Haematologica. 2018;103(12):2088-96.

12. Minarik J, Pour L, Maisnar V, Spicka I, Jungova A, Jelinek T, et al. Single agent daratumumab in advanced multiple myeloma possesses significant efficacy even in an unselected "real-world" population. Biomed Pap Med Fac Univ Palacky Olomouc Czech Repub. 2018. https://doi.org/10.5507/bp.2018.064.

13. Jullien M, Trudel S, Tessoulin B, Mahé B, Dubruille V, Blin N, et al. Single-agent daratumumab in very advanced relapsed and refractory multiple myeloma patients: a real-life single-center retrospective study. Ann Hematol. 2019. https://doi.org/10.1007/ s00277-019-03655-5.

14. Richardson PG, San Miguel JF, Moreau P, Hajek R, Dimopoulos MA, Laubacj JP, et al. Interpreting clinical trial data in multiple myeloma: translating findings to the real-world setting. Blood Cancer J. 2018;8(11):109.

Publisher's Note Springer Nature remains neutral with regard to jurisdictional claims in published maps and institutional affiliations. 